\title{
Inspeção visual do colo uterino após aplicação de ácido acético no rastreamento das neoplasias intra-epiteliais e lesões induzidas por HPV
}

\author{
Cervical visual inspection after application of acetic acid in screening \\ intraepithelial neoplasia and HPV-induced lesions
}

\begin{abstract}
Maria Rachel Aguiar Cordeiro을 Hélio de Lima F. Fernandes Costa $^{2}$, Rosângela Pontes de Andrade ${ }^{3}$, Virginia Ribes Amorim Brandão ${ }^{4}$, Raquel Santana ${ }^{5}$
\end{abstract}

\section{RESUM0}

Objetivo: estimar a validade da inspeção visual após aplicação de ácido acético (IVA) no rastreamento das neoplasias intraepiteliais cervicais (NIC) e lesões induzidas por HPV, comparando seu desempenho com o da colpocitologia e da colposcopia. Métodos: estudo de validação de teste diagnóstico realizado em 893 mulheres de 18 a 65 anos, rastreadas simultaneamente com colpocitologia, IVA e colposcopia, em unidade de saúde pública de Recife, PE. A IVA foi realizada por embrocação do colo com ácido acético a 5\% e observação a olho nu, com auxílio de foco clínico comum. Considerou-se como positividade o achado de qualquer lesão aceto-branca no colo. O padrão-ouro foi o histopatológico de biópsia cervical, realizado sempre que qualquer um dos três testes resultasse anormal. Foram estimados e comparados os indicadores de validade de cada teste, com os respectivos intervalos de confiança a 95\%. A concordância entre os resultados dos testes foi avaliada pelo coeficiente kappa $(\kappa)$. Resultados: das 303 mulheres biopsiadas, o estudo histopatológico foi anormal em 24 . Deste total, a IVA foi positiva em 22, conferindo-lhe sensibilidade estimada de $91,7 \%$, especificidade de $68,9 \%$, valor preditivo positivo de $7,5 \%$ e valor preditivo negativo de $99,7 \%$. Comparando-se os intervalos de confiança a 95\%, a IVA mostrou maior sensibilidade que a colpocitologia, mas com menores especificidade e valor preditivo positivo. Houve fraca concordância entre os resultados da IVA e da colpocitologia $(\kappa=0,02)$ e excelente concordância com os da colposcopia $(\kappa=0,93)$. Conclusão: a IVA foi muito mais sensível que a colpocitologia no rastreamento das NIC e lesões HPV-induzidas e teve o mesmo desempenho da colposcopia. Sua baixa especificidade foi responsável por um elevado número de resultados falso-positivos.

PALAVRAS-CHAVE: Inspeção visual com ácido acético; Colposcopia; Colpocitologia; Colo: lesões pré-neoplásicas; Rastreamento; Citologia

\section{ABSTRACT}

Purpose: to estimate the validity of visual inspection of cervical intraepithelial neoplasia (CIN) and HPV-induced lesion screening, after acetic acid application (VIA), and to compare its performance with that of colpocytology and colposcopy. Methods: a diagnostic test validation study involving 893 women aged 18 to 65 years, simultaneously screened with colpocytology, VIA and colposcopy was carried out at a public health unit in Recife, PE. VIA was performed by applying 5\% acetic acid onto the cervix and observing it with the help of a clinical spotlight. The finding of any aceto-white lesion on the cervix was considered positive. The gold standard was the histopathology of cervical biopsy, carried out whenever any of the three test results was abnormal. Validity indicators were estimated for each test, within $95 \%$ confidence intervals. The analysis of agreement between test results was done by the kappa coefficient. Results: of 303 women submitted to biopsy, the histopathological study was abnormal in 24. Among this total, VIA was positive in 22, yielding an estimated $91.7 \%$ sensibility, $68.9 \%$ specificity, and $7.5 \%$ positive predictive value and $99.7 \%$ negative predictive value. Comparing $95 \%$ confidence intervals, VIA was more sensitive than colpocytology, despite a lower specificity and positive predictive value. There was poor agreement between VIA and colpocytology $(\kappa=0.02)$ and excellent agreement with colposcopy $(\kappa=0.93)$. Conclusion: VIA was much more sensitive than colpocytology in the screening of CIN and HPV-induced lesions and presented a performance similar to colposcopy. Its low specificity determined a high number of false-positive results.

KEYWORDS: Visual inspection with acetic acid; Colposcopy; Colpocytology; Cervix: premalignant lesions; Cancer: screening; Cytology

Centro Integrado de Saúde Amaury de Medeiros - CISAM.

Departamento Materno Infantil da Faculdade de Ciências Médicas - Universidade de Pernambuco - UPE.

1 Médica Tocoginecologista do CISAM

2 Professor Regente de Tocoginecologia da Faculdade de Ciências Médicas da Universidade de Pernambuco (UPE).

3 Médica Patologista do CISAM

4 Professora Assistente da Disciplina de Patologia da Universidade de Pernambuco - UPE

5 Acadêmica de Medicina da Faculdade de Ciências Médicas da Universidade de Pernambuco - UPE

Correspondência: Maria Rachel Aguiar Cordeiro

Rua Jonathas de Vasconcelos, 500, apt. 402 - Boa Viagem - 51021-140 - Recife - PE - Fones: (81) 3467-9994, 3426-2111, Fax: 3426-2111

e-mail: pmbgf@terra.com.br

Recebido em: 5/11/2004

Aceito com modificações em: 24/1/2005 
Introdução

O câncer do colo do útero constitui verdadeiro problema de saúde pública nos países em desenvolvimento $^{1}$, onde programas organizados de rastreamento inexistem ou são esporádicos e de baixa qualidade ${ }^{2-4}$. Menos de cinco por cento da população feminina destes países consegue ser adequadamente rastreada, usualmente em clinicas privadas e em alguns centros urbanos, beneficiando geralmente as de mais baixo risco para a doença ${ }^{5,6}$. Como resultado, a grande maioria dos casos é diagnosticada em fase avançada, com custos terapêuticos elevados e baixa possibilidade de cura, gerando repercussões socioeconômicas e psicológicas importantes ${ }^{6}$. Na tentativa de superar as falhas e obstáculos inerentes ao programa de rastreamento citológico, pesquisadores em todo o mundo têm dado especial atenção ao desenvolvimento de métodos de rastreio alternativos, em especial para os locais onde a população apresenta maiores restrições econômicas e menor escolaridade ${ }^{7,8}$. A inspeção visual do colo uterino após aplicação de ácido acético (IVA) parece ser um dos mais promissores, pois pode ser realizado por pessoal de saúde não-médico, com redução dos custos com mão-de-obra especializada e ampliação da cobertura da população de alto risco pelo progra$\mathrm{ma}^{9,10}$. Também possui características inerentes a bom método de rastreamento: é simples, de fácil aprendizado, rápido, sensivel, barato e permite a leitura imediata dos resultados, possibilitando a tomada de decisões terapêuticas na mesma visita, o que diminui as perdas de seguimento e abandono de tratamento, problemas muito comuns em locais de poucos recursos ${ }^{7-10}$.

O ácido acético tem ação mucolítica e atua causando desidratação celular e coagulação das proteínas intranucleares, diminuindo a transparência do epitélio, sendo a intensidade do acetobranqueamento diretamente proporcional à gravidade da lesão ${ }^{11}$.

Megevand et al. ${ }^{12}$ e Denny et al. ${ }^{8}$ avaliaram o emprego da IVA em mulheres de alto risco para o câncer cervical em local onde não havia nenhum outro programa de rastreamento disponível e concluíram que o teste foi capaz de diagnosticar mais de sessenta e cinco por cento das lesões intraepitelias escamosas (LIEC) de alto grau e carcinoma invasor. Em locais onde o rastreamento citológico já está implantado, a IVA poderia funcionar como método auxiliar à colpocitologia oncótica, aumentando a sua sensibilidade e melhorando a detecção destas lesões em um terço, sem custos adicionais ${ }^{13,14}$.
Embora a IVA já esteja sendo empregada em várias regiões, em especial na Ásia, África, Índia e América Latina ${ }^{4,10-15}$, sua efetividade no controle e prevenção das neoplasias intra-epiteliais cervicais (NIC) na realidade epidemiológica da nossa região permanece por ser definitivamente estabelecida. Os objetivos do presente estudo são: estimar a validade da IVA realizado por médico no processo de rastreamento das NIC e lesões HPVinduzidas, comparando o seu desempenho com o da colpocitologia oncótica e da colposcopia, bem como estimar a concordância entre os resultados da IVA e os resultados dos dois testes citados.

\section{Métodos}

Estudo de validação de teste diagnóstico realizado em serviço de saúde pública de nível secundário em Recife-PE. Foram incluídas no estudo mulheres que procuraram espontaneamente o serviço de prevenção do câncer do colo uterino da instituição, com idade entre 18 e 65 anos e que aceitaram participar, de maneira livre e esclarecida, da pesquisa. Foram excluídas as gestantes, as histerectomizadas, as com passado de tratamento de câncer cervical e/ou de suas lesões precursoras e as com sangramento genital em atividade.

Participaram do estudo 893 mulheres, atendidas no período compreendido entre 14 de fevereiro e 5 de junho de 2003. A média de idade das mulheres era de 32 anos. Mais de dois terços eram casadas, pertenciam às classes sociais menos favorecidas ( $\mathrm{D}$ e E) e eram usuárias de método contraceptivo hormonal oral. Pouco mais de $60 \%$ tinham dois ou mais filhos e informaram início da atividade sexual e primeiro parto a partir dos 16 anos. 784 mulheres $(87,8 \%)$ haviam realizado o último exame preventivo há menos de três anos, constituindo, portanto, população jovem, regularmente rastreada e de baixo risco para a doença.

Após serem esclarecidas verbalmente da importância da realização do exame preventivo e dos objetivos do trabalho, as mulheres que concordaram em participar do estudo assinaram termo de consentimento e responderam a questionário com dados demográficos e reprodutivos aplicado por entrevistadora. Seqüencialmente, realizaram a coleta de material para colpocitologia oncótica (com três auxiliares de enfermagem do serviço que habitualmente desempenham essa atividade), teste IVA e colposcopia, sendo os dois últimos exames realizados por médico com especialização em colposcopia. 
O teste IVA consistiu de: embrocação de todo colo e vagina com um chumaço de algodão embebido em solução de ácido acético a 5\%. Após um minuto e com o auxílio de foco clínico comum direcionado para a abertura do espéculo, todo o colo era inspecionado em busca de lesões acetorreativas. O teste IVA foi categorizado como positivo quando do aparecimento de qualquer lesão acetobranca, opaca ou brilhante, plana ou sobrelevada, de aspecto verrucoso ou não, no colo. Foi considerado negativo se o colo permanecesse com sua coloração normal (rósea, lisa e uniforme) após aplicação da solução. Os esfregaços citológicos foram corados pela técnica de Papanicolaou e interpretados segundo a classificação do sistema Bethesda de $1988^{16}$ (ainda vigente no serviço por ocasião da coleta dos dados). A colpocitologia oncótica foi categorizada como positiva na presença de células epiteliais escamosas atipicas de significado indeterminado (ASCUS), células epiteliais glandulares atípicas de significado indeterminado (AGUS), LIEC ou carcinoma. Foi considerada negativa na presença de achados normais, distúrbios reativos ou reparativos, inflamação e infecção não relacionadas ao HPV. Foi categorizada como insatisfatória em nove pacientes: quatro por apresentarem-se com cervicite intensa, duas com celularidade do esfregaço insuficiente para avaliação citológica, duas com esfregaços dessecados e uma com esfregaço bastante espesso e purulento. A colposcopia foi descrita em formulário padronizado do serviço e obedeceu às orientações do Comitê Internacional de Colposcopia ${ }^{17}$, tendo sido considerada positiva na presença de achados colposcópicos anormais ou sugestivos de câncer cervical invasor. Foi categorizada como negativa se presentes achados colposcópicos normais e como insatisfatória se a junção escamo-colunar (JEC) ou o colo não eram visualizados e na presença de inflamação e/ou atrofia intensas.

Caso qualquer um dos três exames resultasse positivo, a mulher era submetida à biópsia cervical dirigida pela colposcopia. O material assim obtido era enviado para estudo com médico patologista da Unidade, sendo os resultados histopatológicos descritos de acordo com a classificação histológica de Richart $^{18}$. O exame histopatológico foi considerado o padrão-ouro da pesquisa, sendo categorizado como positivo na presença de infecção por HPV, NIC (com ou sem infecção por HPV associada) ou carcinoma invasor. Foi considerado negativo na presença de processo inflamatório inespecífico e na ausência de células atípicas. Também foi inferido como resultado negativo do padrão-ouro quando o exame histopatológico não foi realizado devido à negatividade de todos os testes estudados. Foi considerado insatisfatório em duas pacientes por quantidade insuficiente do material enviado para avaliação histológica.

Todos os profissionais envolvidos na pesquisa desconheciam os resultados dos exames anteriormente realizados. Estimaram-se a acurácia, sensibilidade, especificidade, valor preditivo positivo e valor preditivo negativo de todos os testes de rastreamento do estudo. Comparou-se o desempenho de cada teste pela identificação da sobreposição, ou não, dos intervalos de confiança a $95 \%$ dos indicadores de validade de cada um deles. Procedeu-se à avaliação da concordância entre os resultados da IVA e os resultados de cada método de rastreio pelo cálculo do índice kappa.

O presente trabalho foi aprovado pelo Comitê de Ética do Centro Integrado de Saúde Amaury de Medeiros (CISAM) antes do início da coleta dos dados.

\section{Resultados}

Do total de mulheres do estudo ( $n=893), 303$ foram biopsiadas, sendo o resultado do exame histopatológico considerado anormal em 24 pacientes $(2,7 \%)$ : $14(1,6 \%)$ compativeis com infecção pelo HPV e $11(1,1 \%)$ casos de NIC, sendo 5 casos de NIC I, 3 de NIC II e 2 de NIC III (Tabela 1). Ao avaliar os resultados da colpocitologia oncótica, o exame foi considerado negativo em 878 mulheres $(99,3 \%)$, sendo $269(30,1 \%)$ esfregaços normais e $609(68,2 \%)$ com alterações citológicas benignas (reativas ou reparativas). A colpocitologia foi positiva em $6(0,7 \%)$ mulheres, sendo $1(0,1 \%)$ caso de ASCUS, $4(0,5 \%)$ casos de LIEC baixo grau e $1(0,1 \%)$ de alto grau. O exame colpocitológico foi considerado insatisfatório para avaliação em 9 (1,0\%) mulheres.

Tabela 1- Distribuição das mulheres submetidas a exames preventivos do câncer cervical, de acordo com os resultados dos exames histopatológicos.

\begin{tabular}{lrr}
\hline Resultado da histopatologia & \multicolumn{1}{c}{$\mathbf{n}$} & \multicolumn{1}{c}{$\%$} \\
\hline Negativo para neoplasia & 277 & 31,0 \\
Compatível com HPV & 14 & 1,6 \\
NIC I - Displasia leve & 5 & 0,6 \\
NIC II - Displasia moderada & 3 & 0,3 \\
NIC III - Displasia acentuada / carcinoma in situ & 2 & 0,2 \\
Carcinoma/Adenocarcinoma & 0 & - \\
Insatisfatório & 2 & 0,2 \\
Não realizado & 590 & 66,1 \\
Total & 893 & 100,0 \\
\hline NIC = neopasas intropitliais cervicais & &
\end{tabular}

$\mathrm{NIC}=$ neoplasias intra-epiteliais cervicais. 
A colposcopia foi considerada anormal em 294 (32,9\%) mulheres, negativa em 526 (58,9\%) e insatisfatória por não visualização da JEC em $73(8,2 \%)$.

A IVA foi positiva em 292 das 893 mulheres $(32,7 \%)$ e negativa em $601(67,3 \%)$. Os resultados dos testes de rastreamento (IVA, colpocitologia e colposcopia) foram categorizados dicotomicamente em positivos e negativos. Foi testada a associação dos resultados de cada método de rastreamento com os resultados do padrão-ouro (exame histopatológico), constatando-se uma associação estatisticamente significante $(\mathrm{p}<0,0001)$ deste último com todos os métodos (Tabela 2).
Dos 24 resultados histopatológicos anormais, a IVA foi positiva em 22, conferindo-lhe sensibilidade estimada de 91,7\% (IC 95\% 71,5-98,5\%). Dos histopatológicos negativos, o resultado da IVA foi falso-positivo em 31,1\%. A colpocitologia oncótica identificou corretamente apenas 5 dos 24 histopatológicos anormais, apresentando uma sensibilidade de 20,8\% (IC95\% 7,9\%-42,7), porém uma especificidade de quase $100 \%$. A colposcopia identificou todos os histopatológicos anormais $(\mathrm{S}=$ 100,0\%; IC 95\% 92,8-100,0\%) e apresentou resultado falso-positivo em quase $34 \%$ das mulheres sem anormalidades no histopatológico cervical (Tabela 3).

Tabela 2 - Distribuição das mulheres submetidas a exames preventivos do câncer cervical, de acordo com os resultados dos testes de rastreamento e do histopatológico do colo uterino.

\begin{tabular}{|c|c|c|c|c|c|c|c|}
\hline Teste & Com lesão* & Sem lesão* & $\begin{array}{l}\chi^{2} \\
p\end{array}$ & $\begin{array}{c}S \\
\text { IC } 95 \%\end{array}$ & $\begin{array}{c}E \\
\text { IC } 95 \%\end{array}$ & $\begin{array}{c}\text { VPP } \\
\text { IC } 95 \%\end{array}$ & $\begin{array}{c}\text { VPN } \\
\text { IC } 95 \%\end{array}$ \\
\hline \multicolumn{8}{|l|}{ IVA } \\
\hline Positivo & $22(91,7 \%)$ & $270(31,1 \%)$ & & & & & \\
\hline Negativo & $2(8,3 \%)$ & $599(68,9 \%)$ & 36,26 & $91,70 \%$ & $68,90 \%$ & $7,50 \%$ & $99,70 \%$ \\
\hline Total & $24(100 \%)$ & $869(100 \%)$ & $<0,0001$ & $71,5-98,5$ & $65,7-72,0$ & $4,9-11,3$ & $98,7-99,9$ \\
\hline \multicolumn{8}{|c|}{ Colpocitologia } \\
\hline \multicolumn{8}{|l|}{ Oncótica } \\
\hline Positiva & $5(20,8 \%)$ & $1(0,1 \%)$ & & & & & \\
\hline Negativa & $19(79,2 \%)$ & $859(99,9 \%)$ & $* *$ & $20,80 \%$ & $99,90 \%$ & $83,30 \%$ & $97,80 \%$ \\
\hline Total & $24(100 \%)$ & $860(100 \%)$ & $<0,0001$ & $7,9-42,7$ & $99,2-100$ & $36,5-99,1$ & $96,6-98,7$ \\
\hline \multicolumn{8}{|l|}{ Colposcopia } \\
\hline Positiva & $24(100 \%)$ & $270(33,9 \%)$ & & & & & \\
\hline Negativa & $0(0 \%)$ & $526(66,1 \%)$ & 47,67 & $100,00 \%$ & $66,10 \%$ & $8,20 \%$ & $100,00 \%$ \\
\hline Total & $24(100 \%)$ & $796(100 \%)$ & $<0,0001$ & $92,8-100$ & $62,7-69,3$ & $5,4-12,1$ & $99,1-100,0$ \\
\hline
\end{tabular}

${ }^{*}$ O termo lesão engloba as lesões cervicais HPV-induzidas e as NIC.

- Foram excluídos da análise os casos considerados insatisfatórios.

** Cálculo feito pelo teste exato de Fischer.

$\chi^{2}=$ qui-quadrado.

$\mathrm{S}=$ Sensibilidade, $\mathrm{E}=$ Especificidade, VPP = Valor preditivo positivo, VPN = Valor preditivo negativo; IVA = inspeção visual do colo uterino após aplicação de ácido acétivo.

Tabela 3 - Distribuição das mulheres submetidas a exames preventivos do câncer cervical, de acordo com a concordância dos resultados dos testes de rastreamento e do IVA realizado pelo médico.

\begin{tabular}{|c|c|c|c|c|c|}
\hline \multirow[b]{2}{*}{ Teste de rastreamento } & \multicolumn{2}{|c|}{ IVA (Médico) } & \multicolumn{2}{|c|}{ Concordância com IVA } & \multirow[b]{2}{*}{ Kappa } \\
\hline & Positivo & Negativo & Sim & Não & \\
\hline \multicolumn{6}{|l|}{ Colpocitologia } \\
\hline Positivo & $5(1,7 \%)$ & $1(0,2 \%)$ & $598(67,6 \%)$ & $286(32,4 \%)$ & 0,02 \\
\hline Negativo & $285(98,3 \%)$ & $593(99,8 \%)$ & & & \\
\hline Total & $290(100,0 \%)$ & $594(100,0 \%)$ & & & \\
\hline \multicolumn{6}{|l|}{ Colposcopia } \\
\hline Positiva & $279(95,9 \%)$ & $15(2,8 \%)$ & $793(96,7 \%)$ & $27(3,3 \%)$ & 0,93 \\
\hline Negativa & $12(4,1 \%)$ & $514(97,2 \%)$ & & & \\
\hline Total & $291(100,0 \%)$ & $529(100,0 \%)$ & & & \\
\hline
\end{tabular}

Obs. Foram excluídos do cálculo os resultados considerados insatisfatórios. IVA = inspeção visual so colo uterino após aplicação de ácido acético. 
Os resultados da colpocitologia e da IVA foram concordantes em $67,6 \%$ dos casos e discordantes em 32,4\%, encontrando-se índice kappa igual a 0,02. Quando a IVA foi comparada com a colposcopia, houve resultados discordantes em apenas 3,3\% das mulheres e índice kappa igual a 0,93 (Tabela 3).

\section{Discussão}

Em nossa avaliação, a IVA apresentou sensibilidade superior a $90 \%$. Nossos achados estão condizentes com o de outros pesquisadores, que descreveram em seus estudos que a sensibilidade da IVA oscilaria entre 52 e $96 \%$ e a especificidade entre 49 e $98 \%{ }^{19,20}$. Para estes autores, a sensibilidade do IVA seria diretamente proporcional ao tamanho e gravidade das lesões e inversamente proporcional às alterações epiteliais decorrentes do envelhecimento fisiológico da mulher, as quais tornam a interpretação dos testes de inspeção visual bem mais dificil. A captação do iodo e a cor tornam-se menos intensos com o avançar da idade, em virtude da menor espessura do epitélio da ectocérvice (que fica restrito às suas camadas mais profundas), da tendência à diminuição da zona de transformação e à localização endocervical da JEC ${ }^{21}$. Nos estudos de Denny et al. ${ }^{8}$ e Megevand et al. ${ }^{12}$, enfermeiras treinadas realizaram o exame ginecológico, a IVA e a coleta do esfregaço citológico. Em outros ${ }^{14}$, os mesmos exames foram realizados por citotécnicos. Em todos eles, observaram-se baixa especificidade e elevado número de falso-positivos da IVA ${ }^{7,8,12,14}$. Diferenças nos protocolos de pesquisa, nas populações analisadas, na maneira como o teste é interpretado (os estudos distinguem geralmente os resultados da IVA como positivos ou negativos, sem haver a preocupação com a graduação das lesões) e nas condutas restringem a validade externa dos resultados dos trabalhos já realizados sobre a IVA $^{8,12,14-20}$. Além disso, em alguns deles, há pouca ou nenhuma informação sobre o número de horas de treinamento, número de pacientes vistos ou sobre a qualidade técnica dos instrutores ${ }^{7,8,12}$. Muitas vezes, a iluminação empregada também não é referida ${ }^{8}$ ou é apenas descrita como "adequada"14. A falta de critérios mais rígidos definindo o que realmente seria um teste IVA positivo e a subjetividade na sua avaliação (já que não existe definição universalmente aceita para seus achados) o torna metodologicamente imperfeito, sobrecarregando desnecessariamente os serviços de saúde de referência, já que as taxas de enca- minhamento para colposcopia chegam a 38,7\% e os casos a mais diagnosticados geralmente são de lesões de baixo grau, mais prevalentes e de menor significância clínica ${ }^{22}$.

Adotamos a conduta de encaminhar todas as mulheres para colposcopia, com a biópsia cervical sendo realizada sempre que qualquer um dos testes de rastreamento executados pelo médico resultasse positivo, a fim de minimizar a possibilidade de resultados falso-negativos no padrão-ouro. Assumimos que as mulheres que apresentassem resultado negativo em todos os testes seriam consideradas como verdadeiros negativos do estudo.

Biopsiar todas as mulheres seria antiético, impraticável, demorado e dispendioso. A sensibilidade e a especificidade dos testes estão provavelmente superestimadas, já que os possiveis casos verdadeiros positivos no padrão-ouro e negativos em todos os métodos de rastreio são desconhecidos e, portanto, computados equivocadamente no cálculo dos indicadores de validade. Também excluímos do cálculo todos os casos categorizados como insatisfatórios em cada um dos testes do estudo.

Chock et al. ${ }^{23}$ descreveram um método de cálculo da razão entre os "falso-positivos extras" e os "falso-negativos extras" para comparar a acurácia de dois métodos diagnósticos quando os indivíduos negativos em ambos os testes não se submetem ao padrão-ouro. Seu método, no entanto, pressupõe espectro de gravidade da doença semelhante em populações com diferentes prevalências, pressuposto que não se aplica à doença cervical. Optamos, então, por comparar os indicadores de validade dos métodos pelos seus intervalos de confiança, ressalvando a possível superestimação dos indicadores dada a limitação ética previamente explicitada.

A colpocitologia oncótica, embora seja o exame de rastreamento do câncer cervical mais difundido e aceito em todo o mundo, apresenta imperfeições evidentes ${ }^{24}$. U.ma de suas maiores limitações é a elevada taxa de resultados falso-negativos, com todas as implicações médicas, financeiras e legais dela decorrentes ${ }^{25}$. A maioria de suas falhas resulta de erros na obtenção do esfregaço e apenas um terço, de erros na leitura das lâminas ${ }^{24,26}$. Acreditamos que erros na coleta e o predomínio de mulheres com cervicite em atividade na amostra populacional analisada possam estar relacionados com o elevado número de exames de colpocitologia falsamente negativos que encontramos.

Em nossa pesquisa, a maioria dos resultados anormais da colpocitologia e do estudo histopatológico foi de infecções pelo vírus HPV e NIC I. Esta pode ter sido uma das razões da baixa sensi- 
bilidade estimada para a colpocitologia que, não obstante, situou-se ainda dentro da faixa de 11 a $99 \%$ descrita na literatura ${ }^{27}$. De fato, Nanda et al. ${ }^{26}$ observaram que a sensibilidade da colpocitologia seria diretamente proporcional à gravidade das lesões. A baixa prevalência de lesões pré-neoplásicas encontrada no presente estudo justifica-se pelo fato de a população estudada ser de baixo risco, tendo em vista que mais de $80 \%$ das mulheres analisadas haviam realizado exames preventivos nos últimos três anos.

Resultados de vários estudos já concluídos ou em andamento comparando a IVA e a colpocitologia em um mesmo local apontam para um consenso: o teste IVA é tão ou mais sensivel que a colpocitologia oncótica, embora seja bem menos específico, com taxas de falso-positivos até cinco vezes maiores que as do exame colpocitológico ${ }^{28}$.

Nos países em desenvolvimento, onde coexistem com o problema do câncer cervical, outros de igual magnitude, como as doenças infecciosas, mortalidade infantil e explosão populacional ${ }^{2,6}$, preconiza-se reduzir o número de rastreamentos realizados ao longo da vida da mulher para que se consiga dar maior cobertura ao grupo realmente de risco para a doença. Porém, com esta conduta, a sensibilidade do teste a ser empregado no processo tem que ser elevada, o que não acontece com a colpocitologia oncótica. Uma possibilidade de melhorar a sensibilidade seria pela combinação de testes.

O fato de a IVA e a colposcopia terem sido realizadas seqüencialmente pelo mesmo pesquisador pode ter alterado as estimativas de sensibilidade dos mesmos e ser responsabilizado pela excelente concordância observada entre eles, constituindo importante fonte de viés de detecção.

A reprodutibilidade da IVA pode constituir um problema no rastreamento de rotina, em especial quando realizado por pessoas menos experientes ${ }^{20}$. A mesma limitação se aplica à interpretação dos exames colpocitológicos. Apesar de o objetivo principal de qualquer método de rastreio ser a identificação do maior número possivel de casos verdadeiros da doença, é importante que haja redução dos falso-positivos dos testes a serem empregados, em especial nos locais onde o enfoque "ver e tratar" é adotado. Efeitos colaterais como hemorragia, infecção e estenose cervical, maior risco de contaminação pelo HIV e desconforto para a paciente podem resultar de tratamentos desnecessários que porventura venham a ser realizados ${ }^{14,25}$.

Concluímos que a sensibilidade da IVA para o diagnóstico de NIC e lesões induzidas por HPV foi superior à da colpocitologia, embora esta última tenha se mostrado mais específica e com me- nor número de resultados falso-positivos. Houve ótima concordância entre os resultados da IVA e da colposcopia fraca concordância entre os resultados da IVA e os resultados da colpocitologia.

São necessários estudos posteriores visando familiarizar os prestadores de saúde com os achados deste novo teste, bem como avaliá-lo em local com elevada prevalência da doença e de suas lesões precursoras, a fim de garantir sua reprodutibilidade. Em nossa opinião, é indispensável que seja divulgada e aplicada de maneira uniforme e universal uma padronização dos resultados do teste IVA, e que haja o adequado treinamento e capacitação de todo pessoal envolvido na sua execução, que ela seja supervisionada e com mecanismo de controle de qualidade, visando melhorar sua especificidade e reduzir suas elevadas taxas de exames falsamente positivos, que geram custos financeiros e psicológicos para as pacientes e para o Sistema de Saúde como um todo, antes que seja incorporado como método de rastreio do câncer cervical na prática clínica de nossa realidade.

\section{Referências}

1. Cronjé HS. Screening for cervical cancer in developing countries. Int J Gynaecol Obstet. 2004; 84:101-8.

2. Lazcano-Ponce EC, Castro R, Allen B, Najera P, Alonso de Ruiz PA, Hernández-Avila M. Barriers to early detection of cervical-uterine cancer in Mexico. J Womens Health. 1999; 8:399-408.

3. Denny L. Cervical cancer screening in developing countries. IPPF Med Bull. 2001; 35:3-4.

4. O'Meara AT. Present standards for cervical cancer screening. Curr Opin Oncol. 2002; 14:505-11.

5. Lawson HW, Lee NC, Thames SF, Henson R, Miller DS. Cervical cancer screening among low-income women: results of a national screening program, 1991-1995. Obstet Gynecol. 1998; 92:745-52.

6. Cox RL. Global health disparities: crisis in the diaspora. J Natl Med Assoc. 2004; 96:546-9.

7. Belinson J, Qiao YL, Pretorius R, et al. Shanxi province cervical cancer screening study: a cross sectional comparative trial of multiple techniques to detect cervical neoplasia. Gynecol Oncol. 2001; 83:439-44.

8. Denny L, Kuhn L, Pollack A, Wainwright H, Wright TC Jr. Evaluation of alternative methods of cervical cancer screening for resource-poor settings. Cancer. 2000; 89:826-33. 
9. Cronjé HS, Parham GP, Cooreman BF, de Beer A, Divall P, Bam RH. A comparison of four screening methods for cervical neoplasia in a developing country. Am J Obstet Gynecol. 2003; 188:395-400.

10.Wright TC Jr. Cervical cancer screening using visualization techniques. J Natl Cancer Inst Monogr. 2003; (31):66-71.

11.Franco EL, Duarte-Franco E, Ferenczy A. Prospects for controlling cervical cancer at the turn of the century. Salud Publica Mex. 2003; 45 (Suppl 3):S36775.

12.Megevand E, Denny L, Dehaeck K, Soeters R, Bloch B. Acetic acid visualization of the cervix: an alternative to cytologic screening. Obstet Gynecol. 1996; 88:383-6.

13.Parashari A, Sing V, Sehgal A, Satyanarayana L, Sodhani P, Gupta MM. Low-cost technology for screening uterine cervical cancer. Bull World Health Organ. 2000; 78:964-7.

14.Sankaranarayanan R, Nene BM, Dinshaw K, et al. Early detection of cervical cancer with visual inspection methods: a summary of completed and on-going studies in India. Salud Publica Mex. 2003; 45 (Suppl 3):S399-407.

15.Gaffikin L, Lauterbach M, Blumenthal PD. Performance of visual inspection with acetic acid for cervical cancer screening: a qualitative summary of evidence to date. Obstet Gynecol Surv. 2003; 58:543-50.

16.Solomon D, Davey D, Kurman R, et al. The 2001 Bethesda System: terminology for reporting results of cervical cytology. JAMA. 2002; 287:2114 -9.

17.Stafl A, Wilbanks GD. An international terminology of colposcopy: report of the Nomenclature Committee of the International Federation of Cervical Pathology and Colposcopy. Obstet Gynecol. 1991; $77: 313-4$.

18. Richart RM. A modified terminology for cervical intraepithelial neoplasia. Obstet Gynecol. 1990; 75:131-3.
19.Sankaranarayanan $\mathrm{R}$, Wesley $\mathrm{R}$, Thara S, et al. Test characteristics of visual inspection with $4 \%$ acetic acid (VIA) and Lugol's iodine (VILI) in cervical cancer screening in Kerala, India. Int $\mathrm{J}$ Cancer. 2003; 106:404-8

20.Denny L, Kuhn L, Pollack A, Wright TC Jr. Direct visual inspection for cervical cancer screening: an analysis of factors influencing test performance. Cancer. 2002; 94:1699-707.

21. Mitchell MF, Schottenfeld D, Tortolero-Luna G, Cantor SB, Richards-Kortum R. Colposcopy for the diagnosis of squamous intraepithelial lesions: a meta-analysis. Obstet Gynecol. 1998; 91:626-31.

22. Myers ER, McCrory DC, Subramanian S, et al. Setting the target for a better cervical screening test: characteristics of a cost-effective test for cervical neoplasia screening. Obstet Gynecol. 2000; 96:645-52.

23. Chock C, Irwig L, Berry G, Glaziou P. Comparing dichotomous screening tests when individuals negative on both tests are not verified. J Clin Epidemiol. 1997; 50:1211-7.

24.Basu J, Mikhail M, Palan PR, Payraudeau PH, Romney SL. Factors influencing the exfoliation of cervicovaginal epithelial cells. Am J Obstet Gynecol. 1992; 167:1904-9.

25.Rogstad KE. The psychological impact of abnormal cytology and colposcopy. BJOG. 2002; 109:364-8.

26.Nanda K, McCrory DC, Myers ER, et al. Accuracy of the Papanicolaou test in screening for and followup of cervical cytologic abnormalities: a systematic review. Ann Intern Med. 2000; 132:810-9.

27.Fahey M, Irwig L, Macaskill P. Meta-analysis of Pap test accuracy. Am J Epidemiol. 1995; 141:680-9.

28.Claeys P, De Vuyst H, Gonzalez C, Garcia A, Bello RE, Temmerman M. Performance of the acetic acid test when used in field conditions as a screening test for cervical cancer. Trop Med Int Health. 2003; 8:704-9. 